\title{
Evolution and complexity of micro RNA in the human brain
}

\author{
Walter J. Lukiw* \\ Neurology, Neuroscience and Ophthalmology, Louisiana State University Health Sciences Center, New Orleans, LA, USA \\ ${ }^{*}$ Correspondence: wlukiw@lsuhsc.edu
}

Edited by:

Peng Jin, Emory University School of Medicine, USA

Reviewed by:

Peng Jin, Emory University School of Medicine, USA

The discovery of small non-coding RNAs (sncRNAs), and the biological actions of these ribonucleotide entities in the developing, aging, and pathological human central nervous system (CNS) has opened a novel and fascinating vista into our appreciation of human brain epigenetics, the role of sncRNAs on homeostatic and pathogenic gene control, and the potential role of single stranded, $22 \mathrm{nt}$ signals in shaping the transcriptome of the human CNS (Lukiw et al., 1992; Ambros, 2004, 2011; Lukiw, 2007; Lukiw and Pogue, 2007; Guo et al., 2010). Indeed, a major family of sncRNAs known as micro RNAs (miRNAs) has emerged as essential and critical regulators of gene expression in diverse neurobiological pathways in both health and disease. The primary mode of action of miRNAs is to bind to target ribonucleotide sequences in messenger RNAs (mRNAs), typically in their $3^{\prime}$ un-translated region (3'-UTR), resulting in a highly selective repression of the readout of that mRNA's genetic information (Lukiw, 2007; Guo et al., 2010; Ambros, 2011). Pathogenically up-regulated miRNAs can be considered an epigenetic mechanism to down-regulate specific mRNAs and their expression. Up-regulated miRNA in human brain-specific neurodegenerative disorders such as Alzheimer's disease (AD) may help explain the large number of essential brain gene messages observed to be significantly down-regulated in $\mathrm{AD}$ affected anatomical regions, such as in the temporal lobe neocortex (Brodmann area 22) and hippocampus, or in primary human brain cell cultures that are chronically subjected to $\mathrm{AD}$-relevant stressors (Loring et al., 2001; Colangelo et al., 2002; Lukiw, 2004, 2012; Lukiw and Pogue, 2007; Sethi and Lukiw, 2009; Ginsberg et al., 2012; Lukiw and Alexandrov, 2012; Lukiw et al., 2012; Wang et al., 2012). While the total number of human miRNAs identified to date in all tissues numbers around 2000, the number of abundant human brain miR-
NAs is surprisingly far less; for example only about one-thirtieth of all so far identified human miRNAs are abundantly present in the human brain association neocortex (Table 1). Further, mathematical, bioinformatical, and nucleotide sequence analysis indicate that a $22 \mathrm{nt}$ single stranded RNA composed of four different ribonucleotides (A, G, C, and U) may have as many as $10^{13}$ possible sequence combinations, so the fact that there typically only on the scale of $\sim 10^{2}$ abundant miRNAs in any single human brain sample suggests a very high developmental and evolutionary selection pressure to utilize only specific miRNA oligonucleotide sequences that will yield neurobiologically useful miRNA-mRNA interactions (Lukiw, 2012; Lukiw and Alexandrov, 2012). Table 1 lists the 61 most abundant miRNAs found in a critically controlled pool of short postmortem interval (PMI; $<3 \mathrm{~h}$; total $N=21$ ) human association neocortex (Brodmann area A22) indicating that only about 61 of $10^{13}$, or less than one in one hundred trillion of all potential miRNA species possible have been selected to play some role in brain-essential regulatory functions as a brain abundant miRNA. An additional interesting fact is that miRNAs are highly developmental stage-, tissue- and cell-specific, even in adjacent cell types, and mean abundance of these individual species may fluctuate as do the levels of transcription factors which regulate pre-miRNA transcription (Burmistrova et al., 2007; Lukiw and Pogue, 2007; Guo et al., 2010; Lukiw and Alexandrov, 2012). The stability, and hence persistence of action, of sncRNAs, and miRNA appear to follow the adenine-uracil (AU) element (ARE) rich rules as for mRNA stability, and the half-life of several brain abundant miRNAs has been observed to be surprisingly restricted (Sethi and Lukiw, 2009; Wang et al., 2012). For example, human brain miRNA half-lives have been estimated to range from about $30 \mathrm{~min}$ (miRNA-9) to about $3 \mathrm{~h}$ (miRNA-125b), so overall the generation of a new miRNA, its longevity and interdiction of translational processes may be relatively rapid neurogenetic process (Sethi and Lukiw, 2009). However, other biophysical and neurogenetic parameters such as miRNA secondary and tertiary structure, miRNA binding proteins, and other factors may significantly extend miRNA viability and action.

Another poorly understood layer of complexity in miRNA signaling is that several miRNAs may regulate a single mRNA in a neurogenetic control process known as miRNA convergence, and conversely, a single miRNA may interact with multiple mRNAs in a regulatory process known as miRNA divergence (Lukiw and Alexandrov, 2012). Multiple functioning, and sometimes overlapping, miRNA binding sites have been found, for example, in the $232 \mathrm{nt} 3^{\prime}$-UTR of the human innate immune response regulator complement factor $\mathrm{H}(\mathrm{CFH})$ mRNA in the human brain and retina. Therefore a different repertoire of miRNAs might be used to regulate $\mathrm{CFH}$ expression in either of these related tissue types (Lukiw and Alexandrov, 2012; Lukiw et al., 2012). Indeed, overlapping of miRNA binding sites in the $3^{\prime}$-UTR of the same mRNA may be an evolutionary strategy to regulate specific gene expression via a common genetic signal in multiple and related cell types (Lukiw and Alexandrov, 2012; Lukiw et al., 2012). Anti-miRNA (antagomir, AM) approaches to these multiple miRNA binding sites in mRNA 3'-UTRs are currently redirecting our therapeutic perspectives to potentially yield novel and highly efficacious treatment strategies for brain diseases that have not yet been considered (Lukiw et al., 2008, 2012; Cui et al., 2010; Wang et al., 2012). It will be particularly interesting to analyze global miRNAmRNA targeting and control patterns in the brain and CNS to further unravel this fascinating, evolutionarily selective regulatory 
Table 1 | Most abundant miRNAs in the human brain temporal lobe association neocortex; ranked by miRNA numerical designation; based on pooled data from $N=21$ short post-mortem interval (PMI) controls; this group included 9 males and 12 females, mean age $72 \pm 6.3$ years; all PMls less than $3 \mathrm{~h}$; all of these miRNAs yielded a relative signal strength of 5000 or greater on an LC Sciences miRNA array Genechip (proprietary MRA-1001-miRNA microfluidic chip analytical platform; 1922 small RNAs analyzed; LC Sciences Corporation, Houston, TX, USA) and ranked the top 61 of most abundant miRNAs detected; except for miRNAs listed here, all other miRNAs are in a significantly lower abundance; note high basal abundance of the let-7a to let-7g group of miRNAs; note that AD-abundant miRNAs such as miRNA-155 do not appear in this table as in control brain they are relatively scarce, but are significantly induced as in $A D$ brain, and especially as $A D$ progresses.

\begin{tabular}{ll}
\hline hsa-let-7a-5p & hsa-miR-23b-3p \\
hsa-let-7b-5p & hsa-miR-23c \\
hsa-let-7c & hsa-miR-24-3p \\
hsa-let-7d-5p & hsa-miR-26a-5p \\
hsa-let-7f-5p & hsa-miR-26b-5p \\
hsa-let-7g-5p & hsa-miR-27a-3p \\
hsa-miR-100-5p & hsa-miR-27b-3p \\
hsa-miR-101-3p & hsa-miR-29a-3p \\
hsa-miR-103a-3p & hsa-miR-29b-3p \\
hsa-miR-107 & hsa-miR-29c-3p \\
hsa-miR-124-3p & hsa-miR-30a-5p \\
hsa-miR-125a-5p & hsa-miR-30b-5p \\
hsa-miR-125b-5p & hsa-miR-30c-5p \\
hsa-miR-126-3p & hsa-miR-30d-5p \\
hsa-miR-1273g-3p & hsa-miR-338-3p \\
hsa-miR-128 & hsa-miR-342-3p \\
hsa-miR-139-5p & hsa-miR-3665 \\
hsa-miR-145-5p & hsa-miR-3960 \\
has-miR-146a-5p & hsa-miR-4324 \\
hsa-miR-151a-5p & hsa-miR-4454 \\
hsa-miR-151b & hsa-miR-4516 \\
hsa-miR-16-5p & hsa-miR-451a \\
hsa-miR-181a-5p & hsa-miR-4787-5p \\
hsa-miR-181b-5p & hsa-miR-5001-5p \\
hsa-miR-181d & hsa-miR-5100 \\
hsa-miR-191-5p & hsa-miR-574-3p \\
hsa-miR-195-5p & hsa-miR-9-3p \\
hsa-miR-21-5p & hsa-miR-9-5p \\
hsa-miR-221-3p & hsa-miR-99a-5p \\
hsa-miR-222-3p & hsa-miR-99b-5p \\
hsa-miR-23a-3p & \\
&
\end{tabular}

Note that miRNAs are developmentally and temporally regulated and may display variable abundance in different cells or cell types; larger sample sets need to be analyzed to research these abundance patterns, at this point we cannot exclude the abundance and complexity of other enriched miRNA species. See also Burmistrova et al. (2007).

networking. How individual sncRNA, miRNA, and mRNA complexity together become progressively altered in aging and disease, and their selective quenching using novel AM strategies should be of high therapeutic value in the homeostatic stabilization of miRNA complexity, and in the treatment of common, age-related degenerative disorders, both inside and outside of the central nervous system. and speciation of miRNA in aging, neurodegeneration, Alzheimer's disease (AD), and age-related macular degeneration (AMD) in the Lukiw laboratory were supported in part through Translational Research Initiative (TRI) Grants from LSU Health Sciences Center New Orleans (Walter J. Lukiw), a bioinformatics grant from the Louisiana Biotechnology Research Network (LBRN), an Alzheimer Association InvestigatorInitiated Research Grant IIRG-09-131729 (Walter J. Lukiw), and NIH NIA Grants AG18031 and AG038834 (Walter J. Lukiw). Additional human brain tissues were provided by the Memory Impairments and Neurological Disorders (MIND) Institute and the University of California, Irvine Alzheimer's Disease Research Center (UCIADRC; NIA P50 AG16573). The content of this manuscript is solely the responsibility of the authors and does not necessarily represent the official views of the National Institute on Aging or the National Institutes of Health.

\section{REFERENCES}

Ambros, V. (2004). The functions of animal microRNAs. Nature 431, 350-355.

Ambros, V. (2011). MicroRNAs and developmental timing. Curr. Opin. Genet. Dev. 21, 511-517.

Burmistrova, O. A., Goltsov, A. Y., Abramova, L. I., Kaleda, V. G., Orlova, V. A., and Rogaev, E. I. (2007). MicroRNA in schizophrenia: genetic and expression analysis of miR-130b (22q11). Biochemistry Mosc. $72,578-582$.

Colangelo, V., Schurr, J., Ball, M. J., Pelaez, R. P., and Lukiw, W. J. (2002). Gene expression profiling of 12633 genes in Alzheimer hippocampal CA1: transcription and neurotrophic factor down-regulation and up-regulation of apoptotic and pro-inflammatory signaling. J. Neurosci. Res. 70, 462-473.

Cui, J. G., Li, Y. Y., Zhao, Y., Bhattacharjee, S., and Lukiw, W. J. (2010). Differential regulation of interleukin-1 receptor-associated kinase-1 (IRAK-1) and IRAK-2 by miRNA-146a and NF-kB in stressed human astroglial cells and in Alzheimer disease. J. Biol. Chem. 285, 38951-38960.

Ginsberg, S. D., Alldred, M. J., and Che, S. (2012). Gene expression levels assessed by CAl pyramidal neuron and regional hippocampal dissections in Alzheimer's disease. Neurobiol. Dis. 45, 99-107.

Guo, H., Ingolia, N. T., Weissman, J. S., and Bartel, D. P. (2010). Mammalian microRNAs predominantly act to decrease target mRNA levels. Nature 466, 835-840.

Loring, J. F., Wen, X., Lee, J.M., Seilhamer, J., and Somogyi, R. (2001). A gene expression profile of Alzheimer's disease. DNA Cell Biol. 20, 683-695.

Lukiw, W. J. (2004). Gene expression profiling in fetal, aged, and Alzheimer hippocampus: a continuum of stress-related signaling. Neurochem. Res. 29, 1287-1297.

Lukiw, W. J. (2007). Micro-RNA speciation in fetal, adult and Alzheimer's disease hippocampus. Neuroreport $18,297-300$. 
Lukiw, W. J. (2012). NF-KB-regulated micro RNAs (miRNAs) in primary human brain cells. Exp. Neurol. 235, 484-490.

Lukiw, W. J., and Alexandrov, P. N. (2012). Regulation of complement factor $\mathrm{H}$ (CFH) by Multiple miRNAs in Alzheimer's disease (AD) brain. Mol. Neurobiol. PMID: 22302353. [Epub ahead of print].

Lukiw, W. J., Handley, P., Wong, L., and McLachlan, D. R. C. (1992). BC200 and other small RNAs in normal human neocortex, non-Alzheimer dementia (NAD), and senile dementia of the Alzheimer type (AD). Neurochem. Res. 17, 591-597.

Lukiw, W. J., and Pogue, A. I. (2007). Induction of specific miRNA species by ROS-generating metal sulfates in primary human brain cells. J. Inorg. Biochem. 101, 1265-1269.
Lukiw, W. J., Surjyadipta, B., Dua, P., and Alexandrov, P. N. (2012). Common micro RNAs (miRNAs) target complement factor $\mathrm{H}(\mathrm{CFH})$ regulation in Alzheimer's disease $(\mathrm{AD})$ and in age-related macular degeneration (AMD). Int. J. Biochem. Mol. Biol. 3, 105-116.

Lukiw, W. J., Zhao, Y., and Cui, J. G. (2008). An NF-kBsensitive micro RNA-146a-mediated inflammatory circuit in Alzheimer disease and in stressed human neural cells. J. Biol. Chem. 283, 31315-31322.

Sethi, P., and Lukiw, W. J. (2009). Micro-RNA (miRNA) abundance and stability in human primary brain cells and in human brain tissues. Neurosci. Lett. 459, 100-104.

Wang, H., Chiu, M., Xie, Z., Chiu, M., Liu, Z., Chen, P., Liu, S., Byrd, J. C., Muthusamy, N., Garzon, R., Croce, C. M., Marcucci, G., and Chan, K. K. (2012). Synthetic
microRNA cassette dosing: pharmacokinetics, tissue distribution and bioactivity. Mol. Pharm. 9, 1638-1644

Received: 27 July 2012; accepted: 13 August 2012; published online: 03 September 2012

Citation: Lukiw WJ (2012) Evolution and complexity of micro RNA in the human brain. Front. Gene. 3:166. doi: 10.3389/fgene.2012.00166

This article was submitted to Frontiers in Non-Coding RNA, a specialty of Frontiers in Genetics.

Copyright (c) 2012 Lukiw. This is an open-access article distributed under the terms of the Creative Commons Attribution License, which permits use, distribution and reproduction in other forums, provided the original authors and source are credited and subject to any copyright notices concerning any third-party graphics etc. 